\title{
Neoadjuvant treatments for resectable rectal cancer: A network meta-analysis
}

\author{
WEI ZHONG ${ }^{1}$, XIAOJUN XUE ${ }^{1}$, LIANZHI DAI ${ }^{2}$, RANRAN LI $^{1}, \mathrm{KAINIE}^{1}$ and SONG ZHOU $^{1}$ \\ ${ }^{1}$ Department of General Surgery; ${ }^{2}$ Medical Affairs Department, \\ The Affiliated Southeast Hospital of Xiamen University, Zhangzhou, Fujian 363000, P.R. China
}

Received June 11, 2019; Accepted November 6, 2019

DOI: $10.3892 /$ etm.2020.8494

\begin{abstract}
Different neoadjuvant therapy regimens are available for rectal cancer, but the relative effects are controversial. The aim of the present network meta-analysis (NMA) was to estimate the relative efficacy and safety of neoadjuvant therapies for resectable rectal cancer. MEDLINE, EMBASE and Cochrane Central Registry of Controlled Trials were searched for publications dated from 1946 up to June 2018. The present study included randomized clinical trials that compared treatments for resected rectal cancer: Surgery alone, surgery preceded by neoadjuvant radiotherapy (RT), neoadjuvant chemotherapy (CT) or neoadjuvant chemoradiotherapy (CRT). Direct pairwise comparisons and NMA were conducted. A total of 23 randomized controlled trials were included in the present study. RT had an overall survival (OS) benefit when compared with surgery alone [HR (hazard ratio), $0.89 ; 95 \%$ confidence interval (CI), 0.82-0.97; quality of evidence, high]. All three neoadjuvant regimens were associated with lower local recurrence (LR) when compared with surgery alone [RT: odds ratio (OR), 0.44 ; $95 \%$ CI, 0.35-0.65; quality of evidence, high; CRT: OR, 0.34; 95\% CI, 0.23-0.56; quality of evidence, low and CT: OR, 0.32; 95\% CI, 0.11-1.00; quality of evidence, low]. There were no significant differences in OS and LR between CRT and RT (OS: OR, 1.10); 95\% CI, 0.93-1.20; LR: OR, 0.81; 95\% CI, 0.61-1.10). Ranking probabilities indicated that CRT was the best strategy for local control, with a surface
\end{abstract}

Correspondence to: Dr Song Zhou, Department of General Surgery, The Affiliated Southeast Hospital of Xiamen University, 269 Zhanghua Middle Road, Zhangzhou, Fujian 363000, P.R. China E-mail: 175yyptwk@sina.com

Abbreviations: RT, surgery preceded by neoadjuvant radiotherapy; $\mathrm{CT}$, surgery preceded by neoadjuvant chemotherapy; CRT, surgery preceded by neoadjuvant chemoradiotherapy; OS, overall survival; DFS, disease-free survival; HR, hazard ratio; CI, confidence interval; SUCRA, surface under the cumulative ranking curve; $\mathrm{pCR}$, pathological complete response rate; RCT, randomized clinical trial; NMA, network meta-analysis

Key words: rectal cancer, neoadjuvant, chemoradiation, chemotherapy, network meta-analysis under the cumulative ranking curve (SUCRA) of $78.78 \%$. Patients treated with RT had improved disease-free survival compared with those treated with surgery alone (HR, 0.82; 95\% CI, 0.64-1.00; quality of evidence, low). Neoadjuvant RT or CRT did not significantly improve distant metastases compared with surgery alone (RT: OR, 0.87; 95\% CI, 0.69-1.10 and CRT: OR, 0.75; 95\% CI, 0.47-1.10). CRT had an improved pathological complete response rate compared with RT (OR, 4.90; 95\% CI, 21.80-17.00; quality of evidence, low). No significant difference for the risk of anastomotic leak between each treatment was observed in the NMA. In conclusion, RT decreased the LR and improved OS compared with surgery alone for resected rectal cancer. CRT was the best neoadjuvant therapy analyzed and CT was likely the second best for all outcomes based on SUCRA. However, these findings were limited by overall low quality of evidence.

\section{Introduction}

Rectal cancer represents approximately a third of colorectal cancer cases in the USA and remains an important contributor to the global tumor burden $(1,2)$. The 5-year survival rate of patients with rectal cancer undergoing surgery is $50 \%$ because of the high risk of local recurrence (LR), despite resection being considered as curative (3). The LR of rectal cancer occurs in $30-50 \%$ of patients who have undergone only radical resection (4). Among randomized trials published from 2004 to $2009,30-40 \%$ of patients with rectal cancer developed metastatic rectal cancer (5). With decades of research and practice, important advances have been made in the treatment of rectal cancer with the introduction of total mesorectal excision (TME), the addition of (chemo)radiotherapy preoperatively and the use of magnetic resonance imaging for more accurate clinical staging (6-9).

Improvements in surgical technique have significantly lowered the incidence of rectal cancer LR. Intact removal of the entire mesorectum in cancer of the mid or lower third of the rectum has resulted in LR rates as low as 5-10\% (10). Parallel to improvements in surgical technique, adjuvant therapy regimens have been tested in clinical trials in an effort to improve survival and reduce LR (11). Several randomized controlled trials have evaluated neoadjuvant radiotherapy in patients undergoing resection for rectal cancer and collectively found that it decreased the risk of LR but did not significantly 
improve overall survival (OS) or the rate of distant metastases $(7,12,13)$. However, survival benefit was observed in some trials $(14,15)$.

In order to improve tumor response and long-term survival, preoperative radiotherapy has been combined with chemotherapeutic regimens (16). After long-term exploration and unremitting efforts, preoperative radiotherapy or chemoradiotherapy followed by TME and postoperative chemotherapy is the current recommended regimen for patients with stage II and III rectal cancer (17). Using such multimodality strategies reduces LR rates to $<10 \%$ (11). The addition of neoadjuvant chemotherapy resulted in greater tumor downsizing and downstaging, improved the pathological complete response rate (pCR) and local control, but still led to little OS benefit when compared with preoperative radiotherapy without chemotherapy $(18,19)$. Considering preoperative radiotherapy increases the risk of treatment-related toxicities and the frequency of postoperative complications, some research teams are investigating neoadjuvant chemotherapy without radiotherapy in patients with rectal cancer $(20-22)$. The FOWARC study compared preoperative chemoradiotherapy using folinic acid, fluorouracil and oxaliplatin (FOLFOX) chemotherapy plus radiotherapy, with FOLFOX-based chemotherapy alone. The aforementioned study found that FOLFOX alone seems to have an identical LR rate, 3-year disease-free survival (DFS) and 3-year OS compared to standard FOLFOX plus radiotherapy (20). However, there is still a lack of evidence directly comparing surgery preceded by neoadjuvant chemoradiotherapy (CRT) with surgery preceded by neoadjuvant chemotherapy (CT).

Clinical trials and conventional meta-analysis do not allow comparisons to be made between all regimens, and opinions concerning a definition for optimum neoadjuvant treatment strategy for resected rectal cancer differ (23). Therefore, network meta-analysis (NMA) may be a potential consideration with which to advance the current understanding of the best regimen for resected rectal cancer and to help guide clinical decision making. NMA is a statistical method that aims to combine information from all randomized comparisons between a set of treatments for a given medical condition (24). By using Bayesian NMA in the present study, the objective was to find the most effective neoadjuvant therapy regimen for resected rectal cancer. Regimens were compared in terms of the primary outcomes OS and LR, and the secondary outcomes DFS, distant metastases, pCR, organ preservation, 30-day mortality and anastomotic leak.

\section{Materials and methods}

Literature search. MEDLINE (OvidSP; http://ovidsp. ovid.com/), EMBASE (https://www.embase.com/) and Cochrane Central Registry of Controlled Trials (CENTRAL) (http://cochranelibrary-wiley.com/o/cochrane/clcentral/) were systematically searched in the range between 1946 up to and including May 29 2018. Search terms included extensive controlled vocabulary (medical subject headings and embase subject headings) in various combinations, supplemented with key words including rectal cancer, chemotherapy, radiotherapy, chemoradiotherapy and randomized clinical trials (RCTs). There were no language restrictions made. Electronic searches were performed and supplemented with manual searching for all available articles, including review articles and abstracts from conferences. The literature search strategy used for the present study is depicted in Table SI.

Study selection and data extraction. RCTs that met the following criteria were included in the present study: i) The study enrolled patients with resectable rectal cancer; ii) treatments that administered surgery alone, surgery preceded by neoadjuvant radiotherapy (RT), CT or CRT; and iii) the study reported on at least one of the following outcomes: OS, DFS, perioperative deaths (30-day mortality), pCR, LR, distant metastases, surgery complications and organ preservation. Exclusion criteria included the following: Non-resectable or metastatic rectal cancer, any prior intervention other than diagnostic biopsy and non-randomized trials.

The selection of studies was carried out by two reviewers (WZ and XJX) and included independently screening titles and abstracts for inclusion, extracting the data and assessing the methodological quality of the included studies. Disagreements were resolved by consensus or with a third adjudicator. Data regarding study and population characteristics, as well as treatments and outcomes, were extracted. For OS and DFS, the hazard ratio (HR) and $95 \%$ confidence interval (CI) were extracted when available. If HRs were not reported in the original publications, the HR was calculated using methods outlined by Tierney et al (25). Odds ratios (ORs) were calculated for patterns of recurrence, distant metastases, pCR, organ preservation and surgery complications (for example, perioperative mortality). For multiple reports of the same trial, reports containing the longest follow-up data were used.

Risk of bias assessment. For the included studies, assessment of risk of bias was conducted independently by two reviewers (WZ and XJX) using the Cochrane risk of bias assessment tool $(26,27)$. Studies were assessed on the basis of sequence generation, allocation concealment, blinding, incomplete outcome data, selective outcome reporting and other sources of bias. Any discrepancies were resolved through consensus with a third reviewer (LZD).

Quality of evidence assessment. The quality of evidence for each direct, indirect and NMA outcome was evaluated according to the Grading of Recommendations Assessment, Development and Evaluation method $(28,29)$. The quality of evidence of each direct comparison outcome was ranked as high, moderate, low and very low, based on its risk of bias, consistency, directness, precision of the results and publication bias (28). The quality of evidence of indirect and network effects estimates were derived from those of direct-effects estimates by evaluating network geometry, intransitivity and incoherence (29). For a particular comparison, both direct and indirect evidence were available, the higher of the two quality ratings was presented as the quality rating for the NMA estimate. Detailed information for the quality of evidence of direct and indirect comparisons is shown in Table SII.

Statistical analysis. Evidence for eight outcomes were synthesized: Primary outcomes (OS and LR) and secondary outcomes (DFS, distant metastases, pCR, 30-day mortality, 


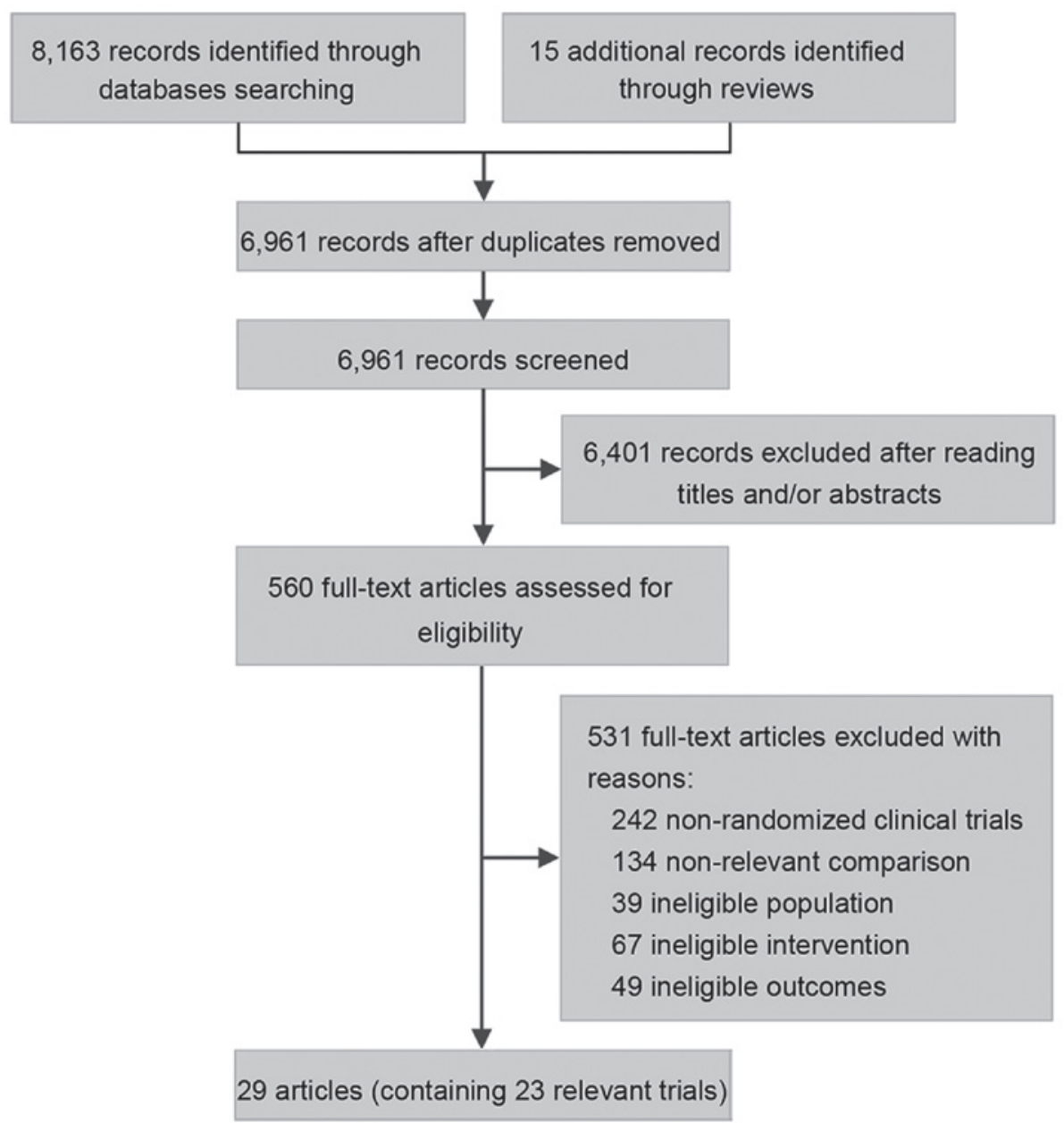

Figure 1. Flow diagram of literature search and article selection process.

anastomotic leak and organ preservation). Direct comparison was performed using a random-effects model to estimate pooled HR or OR and 95\% CI incorporating within- and between-study heterogeneity (30). Statistical heterogeneity of direct comparison was assessed using the $\mathrm{I}^{2}$ index and Cochrane Q test. For each outcome, a Bayesian NMA using Markov chain Monte Carlo simulation with non-informative prior distribution (A prior distribution which is non-committal about a parameter, for example, a uniform distribution) was also performed. The analysis used generalized linear models with a logit link function with 4 chains and 100,000 iterated simulations, discarding the initial 5,000 iterations as burn-in. Convergence was assessed using the Brooks-Gelman-Rubin statistic (31). To test the robustness of this assumption, the node-splitting method was used to assess whether there was incoherence in the closed loop (32).

The Bayesian NMAs also allow for the probabilistic interpretation of uncertainty and ranking of interventions (33). Rank probabilities were calculated from proportions of Markov chain cycles, according to the pooled effect size of each intervention. Surface under the cumulative ranking curve (SUCRA) for each treatment was calculated from a cumulative ranking (34). All the analyses were conducted using $\mathrm{R}$ (version 3.4.1; http://www.R-project.org) with R packages gemtc (version 0.8-2; http://cran.r-project.org/web/packages/gemtc/index.html), meta (version 4.9-5; https://cran.r-project.org/web/packages/meta/index. html) and rjags (vesioin 4-8; https://cran.r-project.org/web/packages/rjags/index.html) and JAGS (version 4.3.0; http://mcmc-jags. sourceforge.net/).

\section{Results}

Study characteristics. Of the 8,178 citations identified via the literature search in the present study, 6,961 citations were retained after removing duplicates and subsequently 6,401 articles were excluded after title and abstract screening, leaving 560 studies for a full-text review. Following exclusion of a further 531 articles which were deemed unsuitable, a total of 23 RCTs with a cumulative sample size of 10,895 patients were included in the review and NMA (7,8,11-14,18-20,35-54; Fig. 1). The number of patients involved in each study ranged from 68-1,805. Of the 23 studies, 15 included trials that made comparisons between RT and surgery alone (7,11-13,35-49). In 6 trials, RT was compared with CRT $(8,14,17-18,54)$. For the two remaining trials, one compared CRT with surgery alone $(50,51)$ and the other compared CRT with CT $(30,31)$. No trial directly comparing CT with RT or surgery alone was included. Details of the baseline characteristics of the included trials and the treatment regimens are presented in Table I.

Risk of bias of included studies. Results of the quality assessment of the trials according to the Cochrane risk-of-bias 


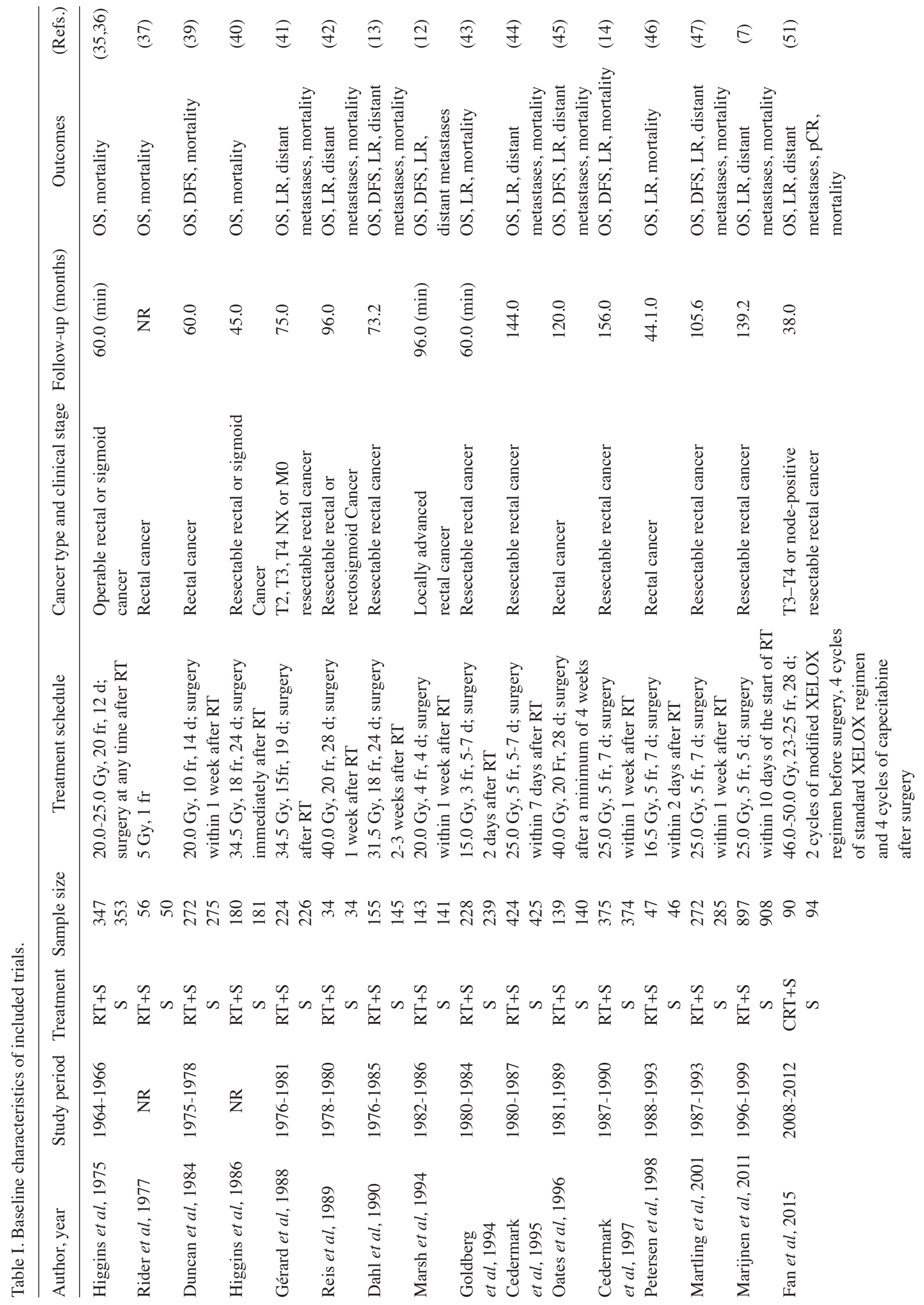




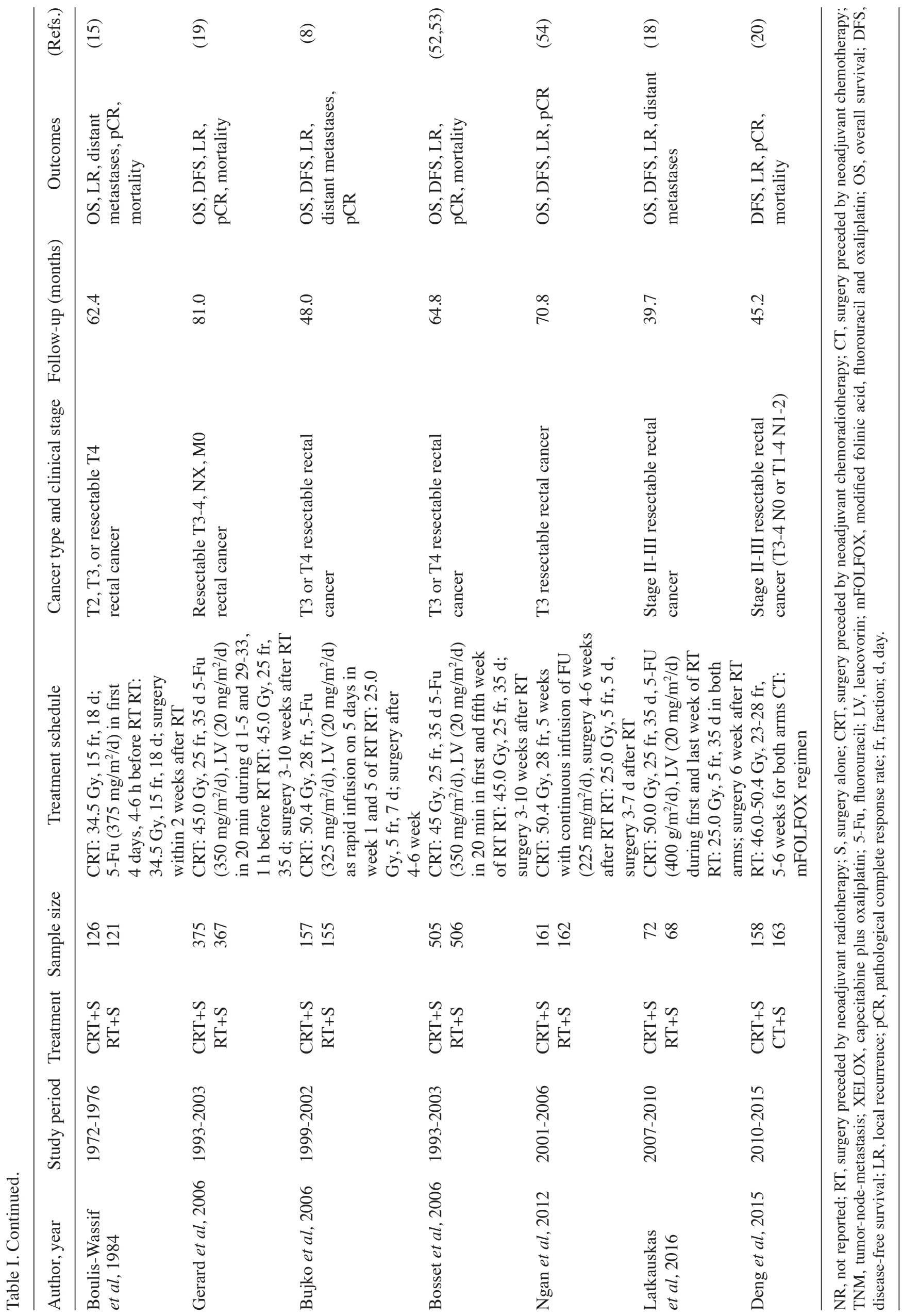


A

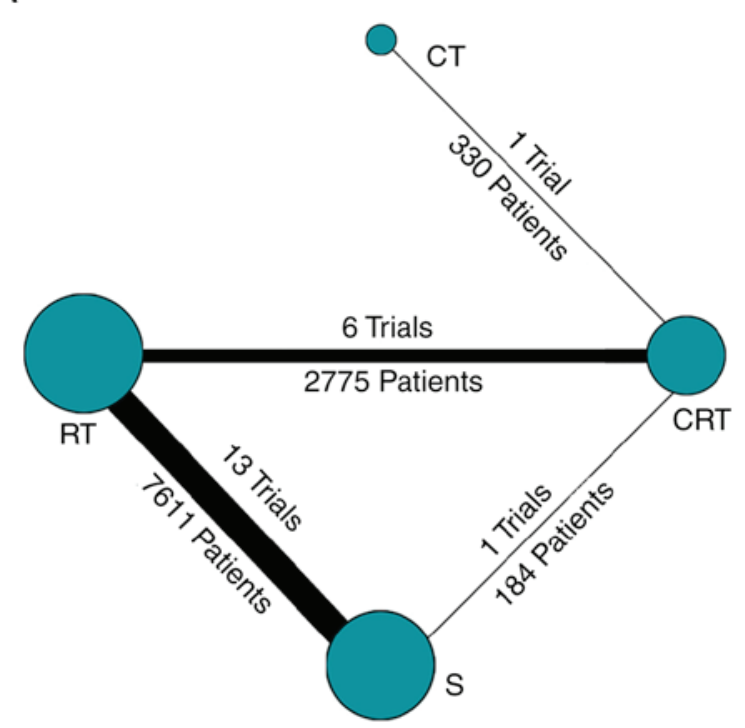

B

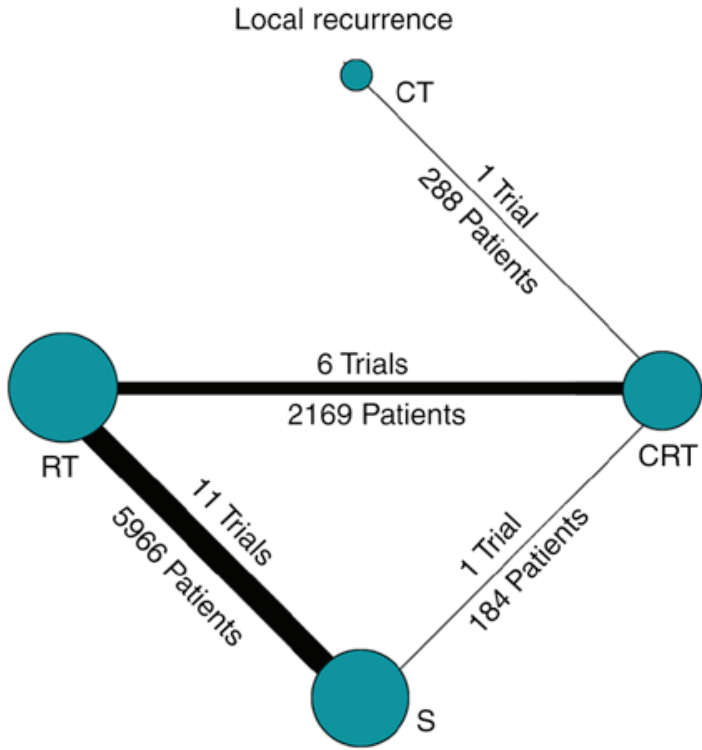

Figure 2. Network geometry for primary outcomes in the network meta-analysis. Each node indicates a strategy and the node size is proportional to the number of patients in the treatment group. Lines represent direct comparisons between two treatments and line thickness represents the number of RCTs included in each comparison, also represented by the numbers. (A) Overall survival (21 trials) and (B) local recurrence (19 trials). S, surgery alone; RT, surgery preceded by neoadjuvant radiotherapy; CRT, surgery preceded by neoadjuvant chemoradiotherapy; CT, surgery preceded by neoadjuvant chemotherapy.

tool (55) are shown in Fig. S1. The overall risk bias was low and was well agreed between reviewers. All the studies included in the NMA were randomized, meaning that the overall selection and attrition bias were minimized. None of the included studies were described as double blind or used blinded outcome assessment. In addition, there were no imbalances between treatment arms in the number of patients that did not undergo the complete trial procedure.

Primary outcome. A total of 21 trials including 10,900 patients and comparing four treatment strategies were included in the 5-year OS analysis (Fig. 2A). As can be seen from pairwise comparison (Table II), RT was associated with improved OS benefit compared with surgery alone (HR, 0.89; 95\% CI, 0.83-0.96; quality of evidence, high). For NMA, there were no significant differences in OS for all comparisons except RT vs. surgery alone (HR, 0.89; 95\% CI, 0.82-0.97; Table II). The SUCRA values of $77.05,56.97$ and $43.57 \%$ for RT, CT and CRT, respectively (Fig. 3A), suggested that these were the three treatments with the highest chance of improving OS in rectal cancer. Sensitivity analyses were also performed with 16 trials for which the total radiation dose $\geq 25 \mathrm{~Gy}$. The results were similar to those for the analysis of all 21 trials.

A total of 19 trials (8,607 patients) comparing three neoadjuvant treatments and surgery were included in the LR analysis (Fig. 2B). The results of NMA suggested a significant advantage of RT or CRT compared with surgery alone (Table II; RT vs. surgery alone: OR, 0.44; 95\% CI, 0.35-0.56; quality of evidence, high and CRT vs. surgery alone: OR, 0.34; 95\% CI, 0.23-0.56; quality of evidence, moderate). There were no significant differences for both direct pairwise analysis and NMA between CRT and RT or CT. Sensitivity analyses were also performed with 15 studies for which the total dose of radiation was $\geq 25 \mathrm{~Gy}$. The results were similar to those for the analysis of all 19 studies. Ranking probabilities analysis further supported the conclusion that CRT and CT were probably the best and second-best strategies, respectively, for local tumor control (Fig. 3B). The SUCRA values of CRT and CT were 78.78 and $74.68 \%$, respectively (Fig. 3B).

Secondary outcome. The NMAs for the 6 secondary outcomes (5-year DFS, distant metastases, pCR, organ preservation, 30-day mortality and anastomotic leak) included 6 to 16 trials involving 2,767 to 7,410 patients with clinically resectable rectal cancer (Fig. S2). The incidence of distant metastases was $28.2 \%$ (1,558 of 5,516), pCR,9.0\% (267 of 2,971), organ preservation $54.0 \%$ (1,496 of 2,767), 30-day mortality $4.1 \%$ (316 of 7,410) and anastomotic leak $5.8 \%$ (175 of 3,042). In DFS analysis, 10 trials comparing the three preoperative treatments were included. The HRs are shown in Table II and Fig. 4. RT was associated with improved DFS than surgery alone (HR, 0.82; 95\% CI, 0.64-1.00; quality of evidence, low). CRT displayed no significant improvement in DFS compared with RT (HR, 0.96; 95\% CI, 0.78-1.20).

The estimated OR of pairwise analysis and NMA for distant metastases, pCR, organ preservation and anastomotic leak are also shown in Table II and Fig. 4. There were no significant differences between interventions for the likelihood of the distant metastases. For trials comparing RT with surgery alone, pCR was not reported and could not be estimated on the basis of the information provided. CRT and CT were associated with improved pCR compared with RT (CRT vs. RT: OR, 4.90; 95\% CI, 1.80-17.00; quality of evidence, low and CT vs. RT: OR, 27.0; 95\% CI, 1.80-530.00; quality of evidence, very low; Fig. 4).

For the analysis of complications, 30-day mortality and anastomotic leak data were extracted. A total of 16 trials (7726 patients) comparing three treatments were included in the 30-day mortality analysis. One trial compared CRT with CT (20), reporting no treatment-related mortality and being 
Table II. Results from direct pairwise comparison and the network meta-analysis.

A, RT vs. S

\begin{tabular}{lcr}
\hline Outcomes & Pairwise comparison & Network meta-analysis \\
\hline Overall survival & HR $0.89(0.83-0.96)^{\mathrm{a}}$ & HR $0.89(0.82-0.97)^{\mathrm{a}}$ \\
Local recurrence & OR $0.44(0.37-0.51)^{\mathrm{a}}$ & OR $0.44(0.35-0.56)^{\mathrm{a}}$ \\
DFS & HR $0.80(0.63-1.02)$ & HR $0.82(0.64-1.00)$ \\
Distant metastases & OR $0.87(0.73-1.05)$ & OR 0.87 (0.69-1.10) \\
Mortality & OR 1.38 $(0.89-2.12)$ & OR $1.40(0.79-2.20)$ \\
pCR & NA & NA \\
Anastomotic leak & OR 1.12 $(0.75-1.67)$ & OR $1.10(0.61-1.80)$ \\
Organ preservation & NA & OR 0.88 $(0.46-1.70)$ \\
\hline
\end{tabular}

\section{B, CRT vs. S}

\begin{tabular}{lcr}
\hline Outcomes & Pairwise comparison & Network meta-analysis \\
\hline Overall survival & HR $1.34(0.43-4.21)$ & HR $0.95(0.81-1.10)$ \\
Local recurrence & OR $1.05(0.25-4.30)$ & OR $0.34(0.23-0.56)$ \\
DFS & HR $1.48(0.34-6.52)$ & HR $0.78(0.58-1.10)$ \\
Distant metastases & OR $0.76(0.30-1.90)$ & OR $0.75(0.47-1.10)$ \\
Mortality & NA & OR 2.10 (0.68-6.40) \\
pCR & NA & NA \\
Anastomotic leak & OR $0.24(0.05-1.18)$ & OR $0.55(0.19-1.50)$ \\
Organ preservation & OR $0.94(0.50-1.77)$ & OR $0.94(0.50-1.80)$ \\
\hline
\end{tabular}

\section{C, CRT vs. CT}

\begin{tabular}{lcc}
\hline Outcomes & Pairwise comparison & Network meta-analysis \\
\hline Overall survival & HR 1.08 $(0.51-2.29)$ & HR $1.10(0.50-2.30)$ \\
Local recurrence & OR 1.06 $(0.46-2.40)$ & OR $1.10(0.46-2.50)$ \\
DFS & NA & NA \\
Distant metastases & NA & NA \\
Mortality & NA & NA \\
pCR & OR $0.18(0.09-0.39)^{\mathrm{a}}$ & OR 0.18 (0.01-2.40) \\
Anastomotic leak & OR 2.58 $(1.25-5.31)^{\mathrm{a}}$ & OR 2.6 (0.83-8.60) \\
Organ preservation & OR 1.24 (0.61-2.51) & OR 1.3 (0.62-2.60) \\
\hline
\end{tabular}

\section{D, CRT vs. RT}

\begin{tabular}{llr}
\hline Outcomes & Pairwise comparison & Network meta-analysis \\
\hline Overall survival & HR 1.06 $(0.92-1.21)$ & HR $1.10(0.93-1.20)$ \\
Local recurrence & OR $0.78(0.48-1.25)$ & OR $0.81(0.61-1.10)$ \\
DFS & HR $0.92(0.82-1.05)$ & HR $0.96(0.78-1.20)$ \\
Distant metastases & OR $0.82(0.51-1.30)$ & OR $0.86(0.57-1.20)$ \\
Mortality & OR $1.55(0.86-2.79)$ & OR $1.60(0.58-4.30)$ \\
pCR & OR 4.01 $(2.24-7.18)^{\mathrm{a}}$ & OR 4.9 $(1.80-17.0)^{\mathrm{a}}$ \\
Anastomotic leak & OR $0.75(0.30-1.85)$ & OR $0.52(0.19-1.30)$ \\
Organ preservation & OR 1.06 $(0.90-1.26)$ & OR $1.10(0.89-1.30)$
\end{tabular}

RT, surgery preceded by neoadjuvant radiotherapy; S, surgery alone; HR, hazard ratio; OR, odds ratio; DFS, disease-free survival; pCR, pathological complete response rate; NA, not applicable; CRT, surgery preceded by neoadjuvant chemoradiotherapy; CT, surgery preceded by neoadjuvant chemotherapy. ${ }^{a} 95 \%$ CI does not contain 1. 
A Overall survival

\begin{tabular}{|lcc|}
\hline & $\begin{array}{c}\text { Ranking, median } \\
(95 \% \mathrm{Cl})\end{array}$ & $\begin{array}{c}\text { Surface under the } \\
\text { cumulative ranking curve }\end{array}$ \\
- CRT & $3(2-4)$ & $43.57 \%$ \\
- CT & $1(1-4)$ & $56.97 \%$ \\
- RT & $2(1-3)$ & $77.05 \%$ \\
- S & $4(2-4)$ & $22.40 \%$ \\
\hline
\end{tabular}

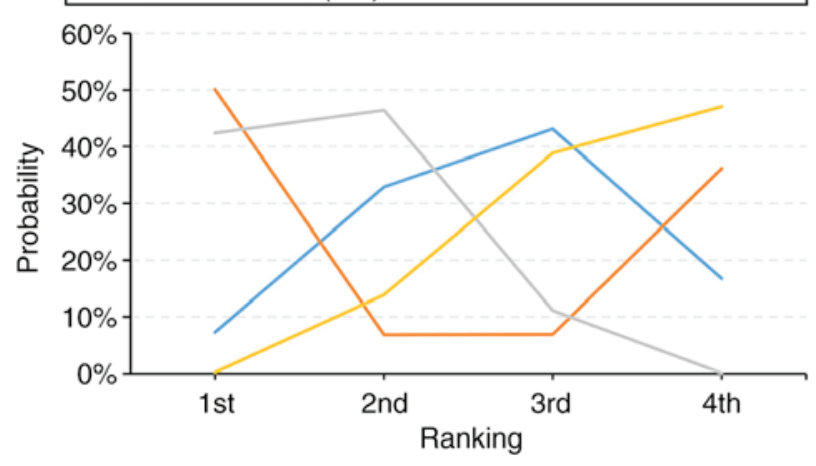

B Local recurrence

\begin{tabular}{|ccc|}
\hline & $\begin{array}{c}\text { Ranking, median } \\
(95 \% \mathrm{Cl})\end{array}$ & $\begin{array}{c}\text { Surface under the } \\
\text { cumulative ranking curve }\end{array}$ \\
- CRT & $2(1-3)$ & $78.78 \%$ \\
- CT & $1(1-3)$ & $74.68 \%$ \\
- RT & $3(1-3)$ & $45.70 \%$ \\
- S & $4(4-4)$ & $0.84 \%$ \\
\hline
\end{tabular}

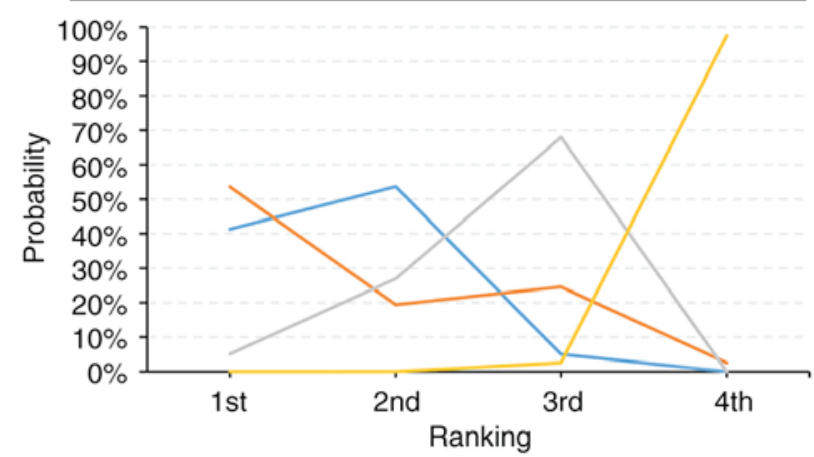

Figure 3. Ranking probability and surface under the cumulative ranking curve of strategies for primary outcomes in the network meta-analysis of neoadjuvant treatments for resectable rectal cancer. Each line represents a treatment strategy. The X-axis indicates the ranking of strategies, with '1st' representing the best. The y-axis represents the probability of each ranking. (A) Overall survival and (B) Local recurrence. S, surgery alone; RT, surgery preceded by neoadjuvant radiotherapy; CRT, surgery preceded by neoadjuvant chemoradiotherapy; CT, surgery preceded by neoadjuvant chemotherapy; CI, confidence interval.
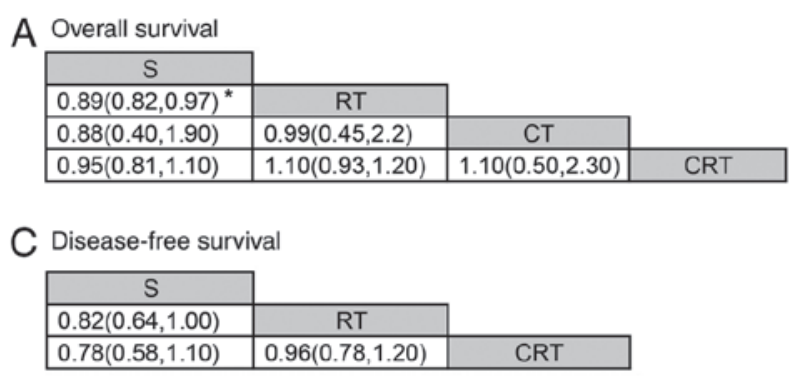

E pCR

\begin{tabular}{|c|c|c|}
\hline RT & & \\
\hline $27(1.80,530){ }^{\star}$ & CT & \\
\hline $4.90(1.80,17)^{*}$ & $0.18(0.013,2.40)$ & CRT \\
\hline
\end{tabular}

B Local recurrence

\begin{tabular}{|l|c|c|c|}
\hline \multicolumn{1}{|c|}{$\mathrm{S}$} & \multicolumn{2}{|c}{} \\
\cline { 1 - 1 } $0.44(0.35,0.56)^{*}$ & $\mathrm{RT}$ & \multicolumn{1}{|c}{} \\
\hline $0.32(0.11,1.00)$ & $0.76(0.31,1.8)$ & $\mathrm{CT}$ & \\
\hline $0.34(0.23,0.56)^{*}$ & $0.81(0.61,1.1)$ & $1.10(0.46,2.50)$ & CRT \\
\hline
\end{tabular}

D Distant metastases

\begin{tabular}{|c|c|c|}
\hline \multicolumn{2}{|c|}{$S$} & \multicolumn{2}{|c}{} \\
\cline { 1 - 1 } $0.87(0.69,1.10)$ & RT & \\
\hline $0.75(0.47,1.10)$ & $0.86(0.57,1.20)$ & CRT \\
\hline
\end{tabular}

F Organ preservation

\begin{tabular}{|c|c|c|c|}
\hline \multicolumn{2}{|c|}{ S } & \multicolumn{2}{|c}{} \\
\cline { 1 - 2 } $0.88(0.46,1.70)$ & RT & \multicolumn{2}{|c}{} \\
\hline $0.75(0.29,2.00)$ & $0.85(0.40,1.80)$ & CT & \\
\hline $0.94(0.50,1.80)$ & $1.10(0.89,1.30)$ & $1.30(0.62,2.60)$ & CRT \\
\hline
\end{tabular}

H 30-day mortality

\begin{tabular}{|c|c|c|}
\hline$S$ & & \\
\hline $1.40(0.79,2.30)$ & RT & \\
\hline $2.20(0.67,6.90)$ & $1.60(0.57,4.50)$ & CRT \\
\hline
\end{tabular}

Figure 4. Results of the network meta-analysis for each outcome for all possible treatment strategies. Effect estimates reflect comparison of the treatment in the row heading vs. the treatment in the column heading. (A) Overall survival presented as hazard ratios (95\% CI). (B) Local recurrence presented as odds ratios (95\% CI). (C) Disease-free survival presented as hazard ratios (95\% CI). (D) Distant metastases, (E) pCR, (F) organ preservation, (G) anastomotic leak and $(\mathrm{H}) 30$-day mortality presented as odds ratios $(95 \% \mathrm{CI})$. CI, confidence interval; pCR, pathological complete response rate; S, surgery alone; RT, surgery preceded by neoadjuvant radiotherapy; CRT, surgery preceded by neoadjuvant chemoradiotherapy; CT, surgery preceded by neoadjuvant chemotherapy. ${ }^{*} 95 \%$ CI does not contain 1.

the only trial including results of CT, this trial was excluded from the NMA. Neoadjuvant strategies (RT and CRT) tended to have more treatment-related mortality than surgery alone, but there were no significant differences (Table II and Fig. 4). The surgical complication of anastomotic leak was reported in nine trials. CT was associated with a lower likelihood of anastomotic leak compared to all other treatments (CT vs. surgery alone: OR, 0.21; 95\% CI, 0.04-0.95; quality of evidence, low; CT vs. RT: OR, 0.20; 95\% CI, 0.04-0.87; quality of evidence, low and CRT vs. CT: OR, 2.60; 95\% CI, 0.83-8.60; quality of evidence, low). The SUCRA analysis suggested that CT had the lowest risk of anastomotic leak with a SUCRA value of $97.26 \%$ (Fig. S3E).

Ranking probability. The ranking probability and the results of SUCRA analysis are shown in Figs. 3 and S3. For the primary outcomes, CT had the highest overall probability of being the best strategy for the neoadjuvant treatment of resectable rectal cancer. Considering all the outcomes, CRT was the best strategy based on SUCRA. 


\section{Discussion}

The NMA in the present study included 23 trials with 10,895 patients with resectable rectal cancer and simultaneously estimated relative effects of four currently used treatment strategies. The present study revealed that preoperative radiotherapy displayed significant survival benefit over surgery alone. No statistically significant differences were observed between CRT and RT or CT. In addition, primary analysis of LR suggested that RT and CRT played a role in local control of rectal cancer when compared with surgery alone, but there were no noticeable differences in survival benefit between the comparisons of three neoadjuvant treatments. With the ranking analysis, CT seemed to be the best strategy among all the included strategies for primary outcomes assessed (CT ranked the first and had the biggest total SUCRA value considering the primary outcomes), whilst CRT was the best strategy when considering all the included outcomes. The evidence for the secondary outcomes was of low quality overall. Out of the outcomes assessed, only anastomotic leak estimated the relative effects of four strategies and CT remained to be the best strategy. The analysis for DFS, distant metastases, pCR and 30-day mortality compared three different strategies, all of them with no significant difference.

Several systemic reviews have evaluated various strategies using conventional pairwise comparison $(3,16,56,57)$. Three meta-analyses confirmed that preoperative radiotherapy improved OS and significantly reduced the LR compared with surgery alone $(3,56,57)$. The present study analyzed the majority of these trials and the results for the primary outcomes were consistent with the results from the previous aforementioned studies. Previously, three systematic reviews have reported that CRT provides no superior OS compared with RT and the result of local control rate was different between these analyses $(16,57,58)$. A previous meta-analysis identified five trials that reported that chemoradiotherapy improved local tumor control as opposed to radiotherapy, with no impact on perioperative outcome or long-term survival (57). The pairwise analysis and NMA carried out in the present study displayed no improvement in both local control and long-term survival for patients treated with CRT compared to those treated with RT. However, significant heterogeneity remained when the data for LR were analyzed using the random effects assumption $\left(\mathrm{I}^{2}=61 \%, \mathrm{P}=0.03\right)$. In this present study, it is unclear whether the heterogeneity is attributable to the addition of chemotherapy to a different RT schedule and a different waiting period until surgery.

To the best of our knowledge, the present study is the first NMA to provide estimates of the outcomes and effects of pairwise comparisons of potential neoadjuvant therapy regimens for resectable rectal cancer. The efficacy of each potential regimen could be ranked using these polled outcomes. However, there was only one eligible trial for CT compared with other strategies (20), which decreased the quality of the whole analysis. Therefore, future studies that directly evaluate survival outcomes and side effects in patients receiving neoadjuvant $\mathrm{CT}$ are warranted.

The present study had some further limitations. Firstly, there were some differences in baseline characteristics of the included trials that could lead to biased results. For example, three of the trials included patients with cancer of the rectosigmoid $(40,41,43)$. The majority of the early trials using Dukes classification included patients from Dukes A-C $(15,32,35)$, whereas previous studies using TNM classification for clinical staging only included patients with stage II/III rectal cancer $(18,20)$. In the MRC I trial, $28 \%$ of patients with metastatic cancer were found to have a Dukes' stage A lesion for which adjuvant therapy is not currently advised (38). These included patients may have led to biased results. Secondly, some regimens with different doses or duration times were grouped together, which may further increase the bias. For example, for the CRT treatment strategy, the total dose of radiation ranged from 34.5-50.4 Gy and three types of chemotherapy regimen (fluorouracil/leucovorin, modified FOLFOX and capecitabine plus oxaliplatin) were used. Thirdly, only one trial compared the neoadjuvant CT regime with CRT therapy strategy, limiting the assessment (20). Fourthly, considering there was no significant survival benefit between the three neoadjuvant treatment strategies, the analysis of the side effects, such as toxicity or quality of life, could be important to evaluate the relative effects of each regime. However, a number of the included trials did not report these outcomes, but future trials should describe them.

Based on the primary and secondary results, the NMA performed in the present study found that neoadjuvant radiotherapy decreases the LR and improves OS compared with surgery alone for resected rectal cancer. Neoadjuvant chemoradiotherapy displayed no significant OS benefit or local control compared with RT. CRT was the best neoadjuvant therapy and CT was likely the second best for all outcomes based on the ranking probability and SUCRA. These findings were limited by overall low quality of evidence.

\section{Acknowledgements}

Not applicable.

\section{Funding}

The present study was supported by the Natural and Scientific grants from Zhangzhou (grant no. ZZ2018J11) and the nursery grants from the Affiliated Southeast Hospital of Xiamen University (grant nos. 16Y007 and 17Y006).

\section{Availability of data and materials}

The datasets used and/or analyzed during the present study are available from the corresponding author on reasonable request.

\section{Authors' contributions}

WZ and SZ contributed to the study design/planning, data collection/entry, data analysis/statistics, data interpretation and funds collection. XJX and LZD contributed to the preparation of the manuscript and literature analysis/search. RRL and KN contributed to the data collection/entry. All authors read and approved the final manuscript. 


\section{Ethics approval and consent to participate}

Not applicable.

\section{Patient consent for publication}

Not applicable.

\section{Competing interests}

The authors declare that they have no competing interests.

\section{References}

1. Siegel RL, Miller KD, Fedewa SA, Ahnen DJ, Meester RGS, Barzi A and Jemal A: Colorectal cancer statistics, 2017. CA Cancer J Clin 67: 177-193, 2017.

2. Stintzing S: Management of colorectal cancer. F1000Prime Rep 6: 108, 2014.

3. Cammà C, Giunta M, Fiorica F, Pagliaro L, Craxì A and Cottone M: Preoperative radiotherapy for resectable rectal cancer: A meta-analysis. JAMA 284: 1008-1015, 2000.

4. Young PE, Womeldorph CM, Johnson EK, Maykel JA, Brucher B, Stojadinovic A, Avital I, Nissan A and Steele SR: Early detection of colorectal cancer recurrence in patients undergoing surgery with curative intent: Current status and challenges. J Cancer 5 : 262-271, 2014

5. Glynne-Jones R and Kronfli M: Locally advanced rectal cancer: A comparison of management strategies. Drugs 71: 1153-1177, 2011.

6. Heald RJ and Ryall RD: Recurrence and survival after total mesorectal excision for rectal cancer. Lancet 1: 1479-1482, 1986

7. van Gijn W, Marijnen CA, Nagtegaal ID, Kranenbarg EM, Putter H, Wiggers T, Rutten HJ, Påhlman L, Glimelius B and Van de Velde CJ; Dutch Colorectal Cancer Group: Preoperative radiotherapy combined with total mesorectal excision for resectable rectal cancer: 12-year follow-up of the multicentre, randomised controlled TME trial. Lancet Oncol 12: 575-582, 2011.

8. Bujko K, Nowacki MP, Nasierowska-Guttmejer A, Michalski W, Bebenek M and Kryj M: Long-term results of a randomized trial comparing preoperative short-course radiotherapy with preoperative conventionally fractionated chemoradiation for rectal cancer. Br J Surg 93: 1215-1223, 2006.

9. MERCURY Study Group: Diagnostic accuracy of preoperative magnetic resonance imaging in predicting curative resection of rectal cancer: Prospective observational study. BMJ 333: 779, 2006.

10. Heald RJ, Moran BJ, Ryall RD, Sexton R and MacFarlane JK Rectal cancer: The Basingstoke experience of total mesorectal excision, 1978-1997. Arch Surg 133: 894-899, 1998.

11. Ceelen WP, Van Nieuwenhove Y and Fierens K: Preoperative chemoradiation versus radiation alone for stage II and III resectable rectal cancer. Cochrane Database Syst Rev CD006041, 2009.

12. Marsh PJ, James RD and Schofield PF: Adjuvant preoperative radiotherapy for locally advanced rectal carcinoma. Results of a prospective, randomized trial. Dis Colon Rectum 37: 1205-1214, 1994.

13. Dahl O, Horn A, Morild I, Halvorsen JF, Odland G, Reinertsen S, Reisaeter A, Kavli H and Thunold J: Low-dose preoperative radiation postpones recurrences in operable rectal cancer. Results of a randomized multicenter trial in western Norway. Cancer 66 : 2286-2294, 1990

14. Swedish Rectal Cancer Trial, Cedermark B, Dahlberg M, Glimelius B, Påhlman L, Rutqvist LE and Wilking N: Improved survival with preoperative radiotherapy in resectable rectal cancer. N Engl J Med 336: 980-987, 1997.

15. Boulis-Wassif S, Gerard A, Loygue J, Camelot D, Buyse M and Duez N: Final results of a randomized trial on the treatment of rectal cancer with preoperative radiotherapy alone or in combination with 5-fluorouracil, followed by radical surgery. Trial of the European organization on research and treatment of cancer gastrointestinal tract cancer cooperative group. Cancer 53: 1811-1818, 1984
16. Onaitis MW, Noone RB, Hartwig M, Hurwitz H, Morse M, Jowell P, McGrath K, Lee C, Anscher MS, Clary B, et al: Neoadjuvant chemoradiation for rectal cancer: Analysis of clinical outcomes from a 13-year institutional experience. Ann Surg 233: 778-785, 2001.

17. National Comprehensive Cancer Network. Rectal cancer (version 2.2018). https://www.nccn.org/professionals/physician_gls/pdf/rectal.pdf. Accessed July, 2018.

18. Latkauskas T, Pauzas H, Kairevice L, Petrauskas A, Saladzinskas Z, Janciauskiene R, Gudaityte J, Lizdenis P, Svagzdys S, Tamelis A and Pavalkis D: Preoperative conventional chemoradiotherapy versus short-course radiotherapy with delayed surgery for rectal cancer: Results of a randomized controlled trial. BMC Cancer 16: 927, 2016.

19. Gérard JP, Conroy T, Bonnetain F, Bouché O, Chapet O, Closon-Dejardin MT, Untereiner M, Leduc B, Francois E, Maurel $\mathrm{J}$, et al: Preoperative radiotherapy with or without concurrent fluorouracil and leucovorin in T3-4 rectal cancers: Results of FFCD 9203. J Clin Oncol 24: 4620-4625, 2006.

20. Deng Y, Chi P, Lan P, Wang L, Cui L, Chen D, Cao J, Wei H, Peng X, Cai G, et al: A multicenter randomized controlled trial of mFOLFOX6 with or without radiation in neoadjuvant treatment of local advanced rectal cancer (FOWARC study): Preliminary results. J Clin Oncol 33, 2015 (In Chinese).

21. Schrag D, Weiser MR, Goodman KA, Gonen M, Hollywood E, Cercek A, Reidy-Lagunes DL, Gollub MJ, Shia J, Guillem JG, et al: Neoadjuvant chemotherapy without routine use of radiation therapy for patients with locally advanced rectal cancer: A pilot trial. J Clin Oncol 32: 513-518, 2014.

22. Zhang J, Huang M, Cai Y, Wang L, Xiao J, Lan P, Hu H, Wu X, Ling J, Peng J, et al: Neoadjuvant chemotherapy with mFOLFOXIRI without routine use of radiotherapy for locally advanced rectal cancer. Clin Colorectal Cancer 18: 238-244, 2019.

23. Lumley T: Network meta-analysis for indirect treatment comparisons. Stat Med 21: 2313-2324, 2002.

24. Rücker G: Network meta-analysis, electrical networks and graph theory. Res Synth Methods 3: 312-324, 2012.

25. Tierney JF, Stewart LA, Ghersi D, Burdett S and Sydes MR: Practical methods for incorporating summary time-to-event data into meta-analysis. Trials 8: 16, 2007.

26. Higgins JP, Altman DG, Gøtzsche PC, Jüni P, Moher D, Oxman AD, Savovic J, Schulz KF, Weeks L and Sterne JA; Cochrane Bias Methods Group; Cochrane Statistical Methods Group: The Cochrane Collaboration's tool for assessing risk of bias in randomised trials. BMJ 343: d5928, 2011.

27. Higgins JP and Altman DG: Assessing Risk of Bias in Included Studies. Cochrane Handbook for Systematic Reviews of Interventions: Cochrane Book Series: 187-241, 2008.

28. Guyatt GH, Oxman AD, Vist GE, Kunz R, Falck-Ytter Y, Alonso-Coello P and Schünemann HJ; GRADE Working Group: GRADE: An emerging consensus on rating quality of evidence and strength of recommendations. BMJ 336: 924-926, 2008.

29. Puhan MA, Schünemann HJ, Murad MH, Li T, Brignardello-Petersen R, Singh JA, Kessels AG and Guyatt GH; GRADE Working Group: A GRADE working group approach for rating the quality of treatment effect estimates from network meta-analysis. BMJ 349: g5630, 2014.

30. DerSimonian R and Laird N: Meta-analysis in clinical trials. Control Clin Trials 7: 177-188, 1986.

31. Brooks SP and Gelman A: General methods for monitoring convergence of iterative simulations. J Comput Graphical Statistics 7: 434-455, 1998.

32. Dias S, Welton NJ, Caldwell DM and Ades AE: Checking consistency in mixed treatment comparison meta-analysis. Stat Med 29: 932-944, 2010

33. Jansen JP, Fleurence R, Devine B, Itzler R, Barrett A, Hawkins N, Lee K, Boersma C, Annemans L and Cappelleri JC: Interpreting indirect treatment comparisons and network meta-analysis for health-care decision making: Report of the ISPOR task force on indirect treatment comparisons good research practices: Part 1. Value Health 14: 417-428, 2011.

34. Salanti G, Ades AE and Ioannidis JP: Graphical methods and numerical summaries for presenting results from multiple-treatment meta-analysis: An overview and tutorial. J Clin Epidemiol 64: 163-171, 2011.

35. Higgins GA Jr, Conn JH, Jordan PH Jr, Humphrey EW, Roswit B and Keehn RJ: Preoperative radiotherapy for colorectal cancer. Ann Surg 181: 624-631, 1975. 
36. Roswit B, Higgins GA and Keehn RJ: Preoperative irradiation for carcinoma of the rectum and rectosigmoid colon: Report of a National Veterans Administration randomized study. Cancer 35: $1597-1602,1975$.

37. Rider WD, Palmer JA, Mahoney LJ and Robertson CT: Preoperative irradiation in operable cancer of the rectum: Report of the Toronto trial. Can J Surg 20: 335-338, 1977.

38. Duncan W, Smith AN, Freedman LS, Alderson MR, Arnott SJ, Bleehen N M, Bond WH, Crowther D, Deeley TJ, Duthie HL, et al: A trial of preoperative radiotherapy in the management of operable rectal cancer. Br J Surg 69: 513-519, 1982.

39. Duncan W, Smith AN, Freedman LS, Alderson MR, Arnott SJ, Bleehen N M, Bond WH, Crowther D, Deeley TJ, Duthie HL, et al: The evaluation of low dose pre-operative X-ray therapy in the management of operable rectal cancer; results of a randomly controlled trial. Br J Surg 71: 21-25, 1984.

40. Higgins GA, Humphrey EW, Dwight RW, Roswit B, Lee LE Jr and Keehn RJ: Preoperative radiation and surgery for cancer of the rectum. Veterans administration surgical oncology group trial II. Cancer 58: 352-359, 1986.

41. Gérard A, Buyse M, Nordlinger B, Loygue J, Pène F, Kempf P, Bosset JF, Gignoux M, Arnaud JP and Desaive C: Preoperative radiotherapy as adjuvant treatment in rectal cancer. Final results of a randomized study of the European organization for research and treatment of cancer (EORTC). Ann Surg 208: 606-614,1988.

42. Reis Neto JA, Quilici FA and Reis JA Jr: A comparison of nonoperative vs. preoperative radiotherapy in rectal carcinoma. A 10-year randomized trial. Dis Colon Rectum 32: 702-710, 1989.

43. Goldberg PA, Nicholls RJ, Porter NH, Love S and Grimsey JE: Long-term results of a randomised trial of short-course low-dose adjuvant pre-operative radiotherapy for rectal cancer: Reduction in local treatment failure. Eur J Cancer 30A: 1602-1606, 1994.

44. Cedermark B, Johansson H, Rutqvist LE and Wilking N: The Stockholm I trial of preoperative short term radiotherapy in operable rectal carcinoma. A prospective randomized trial. Stockholm colorectal cancer study group. Cancer 75: 2269-2275, 1995.

45. Oates G, Stenning S, and Hardcastle J: Randomised trial of surgery alone versus radiotherapy followed by surgery for potentially operable locally advanced rectal cancer. Medical research council rectal cancer working party. Lancet 348: 1605-1610, 1996.

46. Petersen S, Hellmich G, Baumann M, Herrmann T, Henke G and Ludwig K: Brief preoperative radiotherapy in surgical therapy of rectal carcinoma. Long-term results of a prospective randomized study. Chirurg 69: 759-765,1998 (In German).

47. Martling A, Holm T, Johansson H, Rutqvist LE and Cedermark B; Stockholm Colorectal Cancer Study Group: The Stockholm II trial on preoperative radiotherapy in rectal carcinoma: Long-term follow-up of a population-based study. Cancer 92: 896-902, 2001

48. Randomized study on preoperative radiotherapy in rectal carcinoma. Stockholm colorectal cancer study group. Ann Surg Oncol 3: 423-430, 1996.
49. Kapiteijn E, Marijnen CA, Nagtegaal ID, Putter $H$, Steup WH, Wiggers T, Rutten HJ, Pahlman L, Glimelius B, van Krieken JH, et al: Preoperative radiotherapy combined with total mesorectal excision for resectable rectal cancer. N Engl J Med 345: 638-646, 2001.

50. Wang F, Lu ZH, Pan Z, Gao YH, Chen G, Wu X, Ding PR, Zeng ZF and Wan D: Preoperative radiotherapy combined with simultaneous chemotherapy with capecitabine plus oxaliplatin versus surgery alone: A single-centered, phase II study in patients with mid/low rectal cancer. J Clin Oncol 32: 2014 (In Chinese).

51. Fan WH, Wang FL, Lu ZH, Pan ZZ, Li LR, Gao YH, Chen G, Wu XJ, Ding PR, Zeng ZF and Wan DS: Surgery with versus without preoperative concurrent chemoradiotherapy for mid/low rectal cancer: An interim analysis of a prospective, randomized trial. Chin J Cancer 34: 394-403, 2015.

52. Bosset JF, Collette L, Calais G, Mineur L, Maingon P, Radosevic-Jelic L, Daban A, Bardet E, Beny A and Ollier JC; EORTC Radiotherapy Group Trial 22921: Chemotherapy with preoperative radiotherapy in rectal cancer. N Engl J Med 355: 1114-1123, 2006.

53. Bosset JF, Calais G, Mineur L, Maingon P, Radosevic-Jelic L, Daban A, Bardet E, Beny A, Briffaux A and Collette L: Enhanced tumorocidal effect of chemotherapy with preoperative radiotherapy for rectal cancer: Preliminary results--EORTC 22921. J Clin Oncol 23: 5620-5627, 2005.

54. Ngan SY, Burmeister B, Fisher RJ, Solomon M, Goldstein D, Joseph D, Ackland SP, Schache D, McClure B, McLachlan SA, et al: Randomized trial of short-course radiotherapy versus long-course chemoradiation comparing rates of local recurrence in patients with T3 rectal cancer: Trans-tasman radiation oncology group trial 01.04. J Clin Oncol 30: 3827-3833,2012.

55. Higgins JPT, Green S (eds): Cochrane handbook for systematic reviews of interventions, version 5.1.0 (updated Marh 2011). The Cochrane Collaboration 2011.

56. Wong RK, Tandan V, De Silva S and Figueredo A: Pre-operative radiotherapy and curative surgery for the management of localized rectal carcinoma. Cochrane Database Syst Rev CD002102, 2007.

57. Rahbari NN, Elbers H, Askoxylakis V, Motschall E, Bork U, Buchler MW, Weitz J and Koch M: Neoadjuvant radiotherapy for rectal cancer: Meta-analysis of randomized controlled trials. Ann Surg Oncol 20: 4169-4182, 2013.

58. Latkauskas T, Paskauskas S, Dambrauskas Z, Gudaityte J, Saladzinskas S, Tamelis A and Pavalkis D: Preoperative chemoradiation vs radiation alone for stage II and III resectable rectal cancer: A meta-analysis. Colorectal Dis 12: 1075-1083, 2010.

This work is licensed under a Creative Commons Attribution-NonCommercial-NoDerivatives 4.0 International (CC BY-NC-ND 4.0) License. 